\title{
Supporting Information:s
}

16 $\alpha$-Hydroxytrametenolic acid from Poria cocos improves intestinal barrier function through glucocorticoid receptor mediated PI3K/Akt/NF-кB pathway

Hui Xu ${ }^{\mathrm{a}, \mathrm{b}}$, Yuchen Wang ${ }^{\mathrm{c}}$, Peter W. Jurutka ${ }^{\mathrm{d}}$, Yadong Chen ${ }^{\mathrm{c}}$, Chongjiang Cao ${ }^{\mathrm{a}}$, Guitang Chen $^{\mathrm{a}}$, Shen $\mathrm{Wu}^{\mathrm{a}}$, Bei Tian ${ }^{\mathrm{a}}$, Suilou Wang ${ }^{\mathrm{a}^{*}}$ and Shujie Cheng ${ }^{\mathrm{a}^{*}}$

aDepartment of Food Quality and Safety/National R\&D Center For Chinese Herbal Medicine Processing, School of Engineering, China Pharmaceutical University, Nanjing, 211198, China;

${ }^{\mathrm{b}}$ College of Food Science and Engineering/Collaborative Innovation Center for Modern Grain Circulation and Safety/Key Laboratory of Grains and Oils Quality Control and Processing, Nanjing University of Finance and Economics, Nanjing, 210023, China;

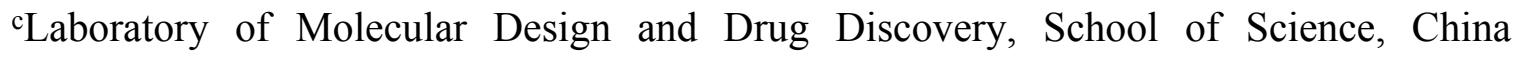
Pharmaceutical University, Nanjing, 211198, China;

dSchool of Mathematical and Natural Sciences, Arizona State University, AZ 85306, USA

* Contact Information of the Corresponding Authors:

Shujie Cheng, E-mail: scheng@cpu.edu.cn

Suilou Wang, E-mail: cpuwsl@126.com 


\section{Supporting figures and tables}

Figure S1. Specificity test of triterpenes from $P$. cocos binding to and activating GR. Caco-2 cells or human 293T cells were transfected with the appropriate firefly luciferase reporter construct and nuclear receptor expression plasmids, as well as Renilla luciferase to normalize for transfection efficiency. Cells were treated with the indicated combinations of ligands for 24 hours and a dual luciferase assay was performed. The cells received an GR expression vector and a luciferase reporter plasmid containing the glucocorticoid responsive element (GRE) derived from the rat tyrosine aminotransferase gene. The results were normalized for transfection efficiency and plotted as a percent of the positive control dexamethasone (Dex)-stimulated transcription (Dex=100\%) in (A) Caco-2 cells and (B) 293 T cells. Each bar represents the mean \pm SD of at least three independent experiments. Mif was used as a GR antagonist. \#\#\# $P<0.001$ vs control group, $* * * P<0.001$ vs Dex group. 

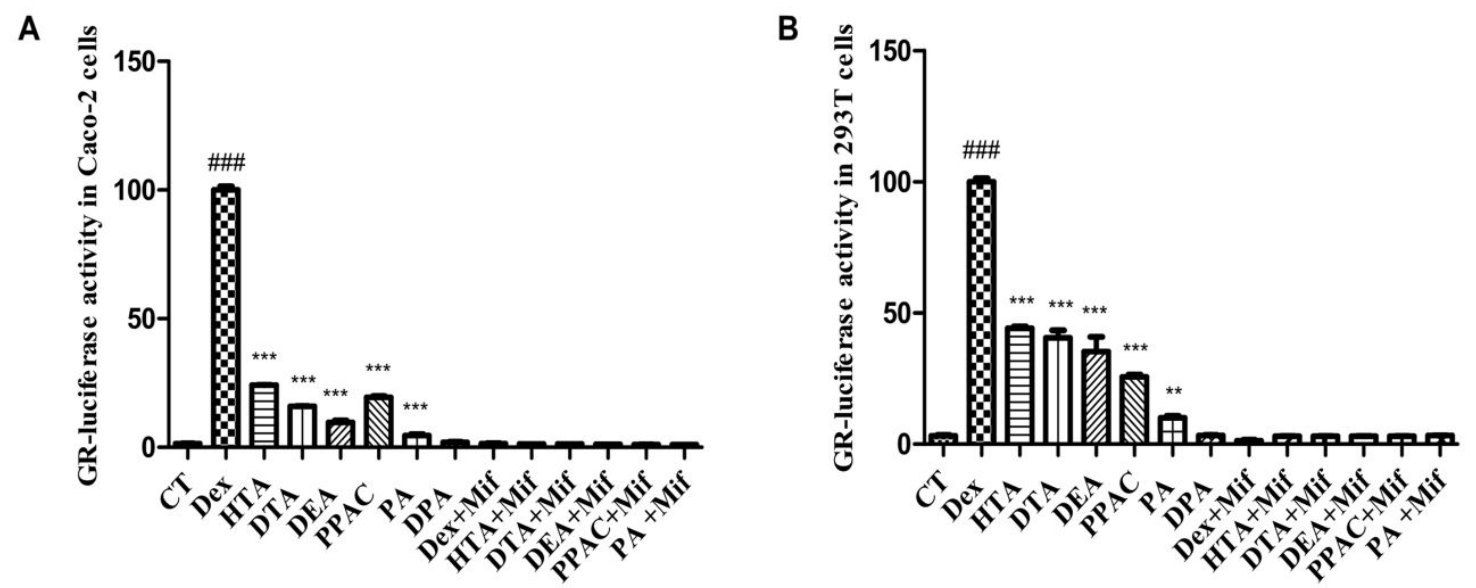

Figure S2. Effect of HTA on TNF- $\alpha$ induced attenuation of TEER. TEER in Caco-2 cell monolayers with HTA $(0-60 \mu \mathrm{M})$ and $\operatorname{Mif}(1 \mu \mathrm{M})$ pretreatment was measured by trans-epithelial voltohmmeter 24 hours after $50 \mathrm{ng} / \mathrm{mL}$ TNF- $\alpha$ administration. Each bar represents the mean $\pm \mathrm{SD}$ of at least three independent experiments. $* * * P<0.001$. 


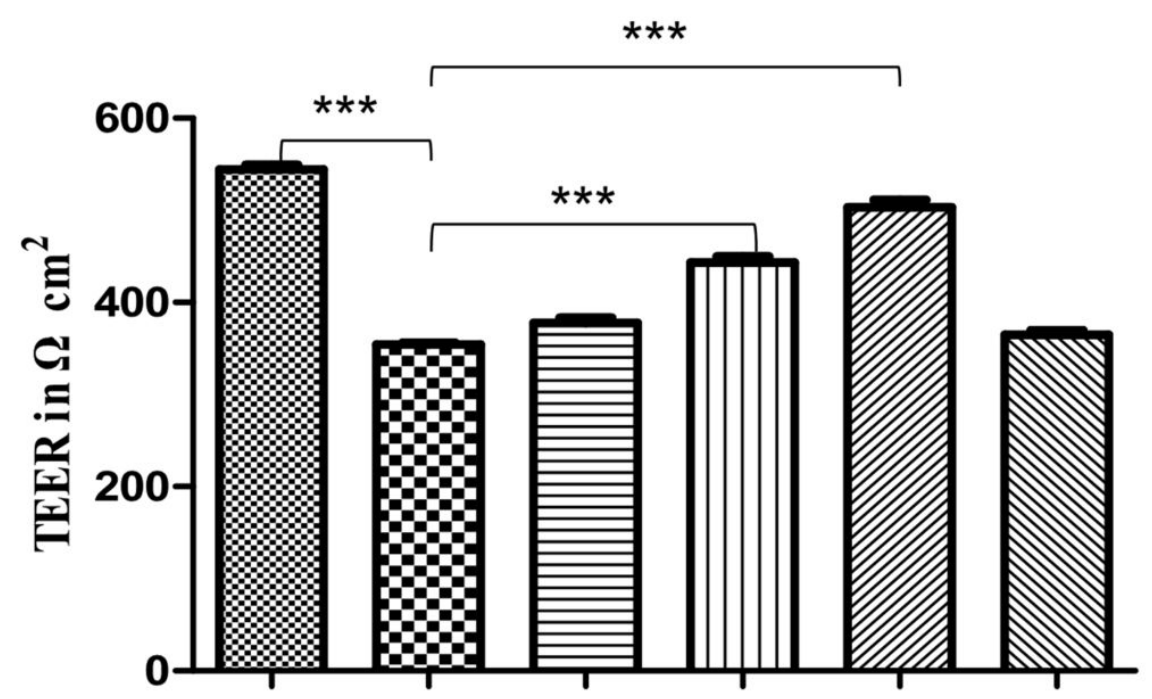

$\begin{array}{lllllll}\text { TNF- } \alpha(\mathrm{ng} / \mathrm{mL}) & 0 & 50 & 50 & 50 & 50 & 50 \\ \text { HTA }(\mu \mathrm{M}) & 0 & 0 & 20 & 40 & 60 & 60 \\ \operatorname{Mif}(\mu \mathrm{M}) & 0 & 0 & 0 & 0 & 0 & 1\end{array}$

Table S1. Chemical structures of triterpenes from Poria cocos. 
1.Trametenolic acid

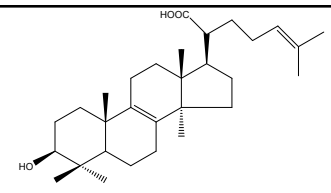

2.Dehydrotrametenolic acid

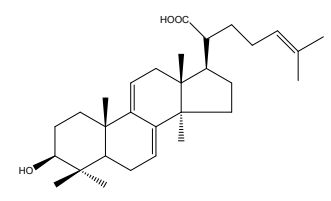

3.3-epi-Dehydrotrametenolic acid

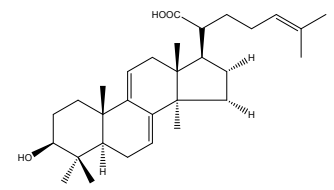

4.16 $\alpha$-Hydroxytrametenolic acid

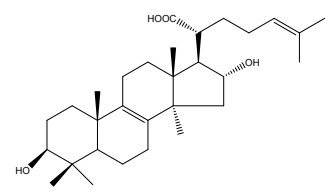

5.3-O-Acetyl-16 $\alpha$-hydroxytrametenolic acid

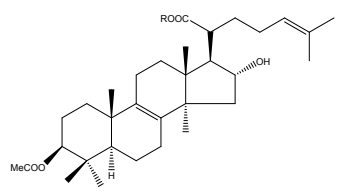

6.Dehydrotrametenonic acid

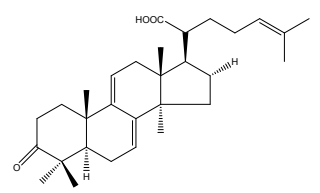

7.3 $\beta, 16 \alpha$-Dihydroxy-lanosta-7,9(11),24-trien-21-

oic acid

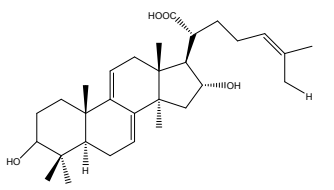

8.Eburicoic acid

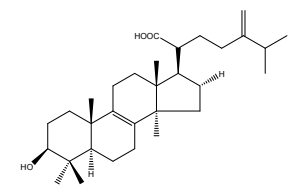


9.Dehydroeburicoic acid

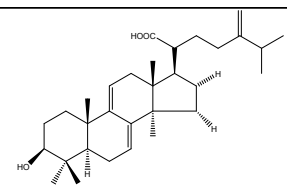

10.16 $\alpha$-25-Dihydroxydehydroeburicoic acid

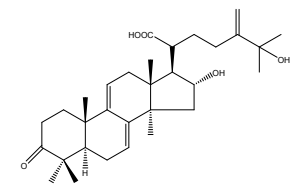

11.Dehydroeburiconic acid

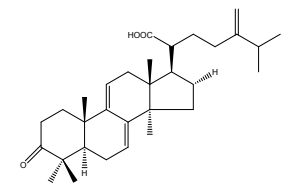

12.16 $\alpha$-Hydroxyeburiconic acid

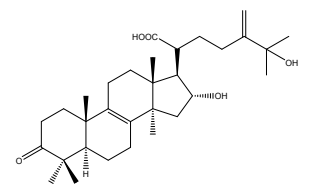

13.16 $\alpha$-25-Dihydroxydehydroeburiconic acid

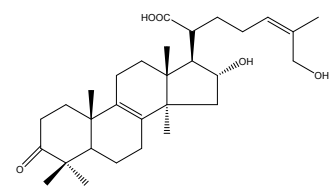

14.Pachymic acid

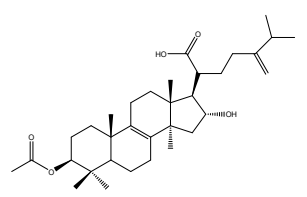

15.Dehydropachymic acid

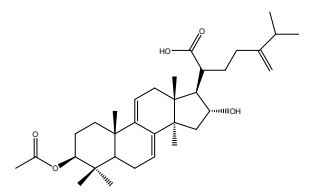

16.3-epi-Dehydropachymic acid

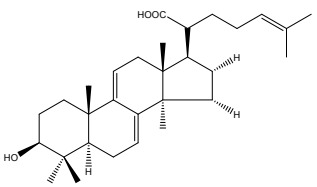


17.16 $\alpha$-Hydroxydehydropachymic acid

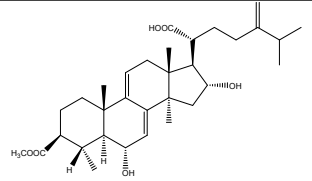

18.25-Hydroxypachymic acid

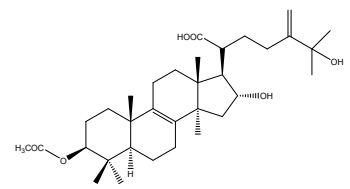

19.Tumulosic acid

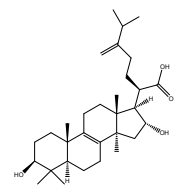

20.Dehydrotumulosic acid

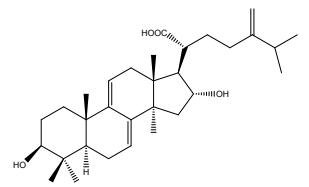

21.3-epi-Dehydrotumulosic acid

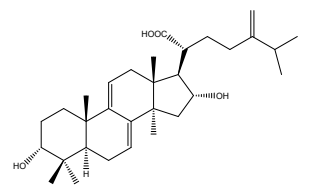

22.15 $\alpha$-Hydroxyl-dehydrotumulosic acid

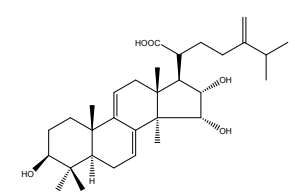

23.25-Hydroxy-3-epi-tumulosic acid

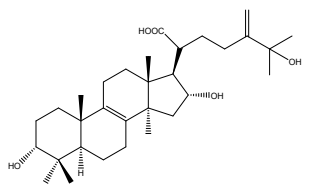

24.25-Hydroxy-3-epi-hydroxytumulosic acid

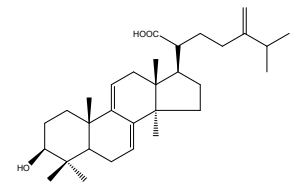


25.33-p-hydroxybenzoyl-dehydrotumulosic acid

$26.5 \alpha, 8 \alpha$-Peroxydehydrotumulosic acid

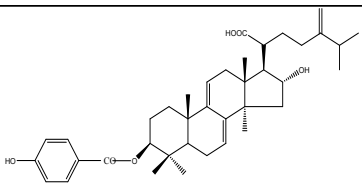

27.Polyporenic acid C

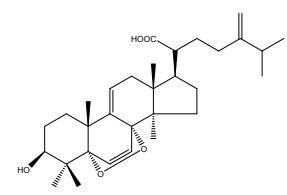

28.6-Hydroxyl-polyporenic acid C

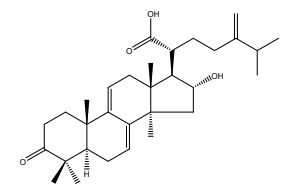

29.29-Hydroxyl-polyporenic acid C

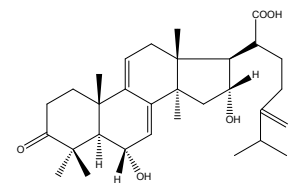

30.Poriacosones A

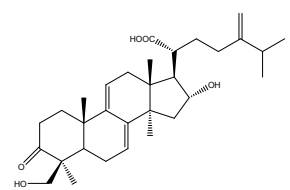

31.Poriacosones B
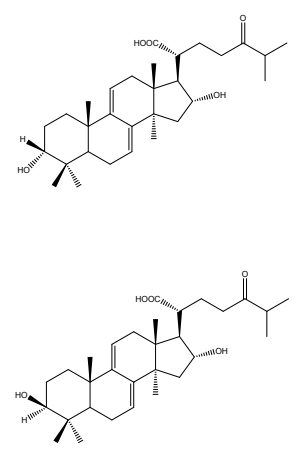

32.Poricoic acid B

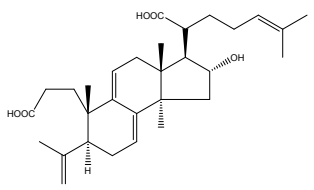


33.16-Deoxyporicoic acid B

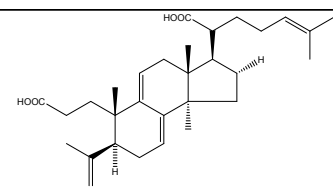

34.Poricoic acid E

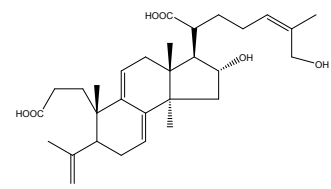

35.Poricoic acid BM

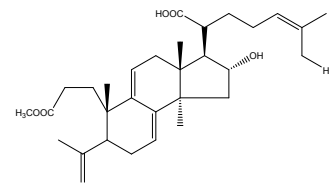

36.Poricoic acid G

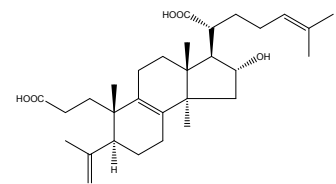

37.Poricoic acid GM

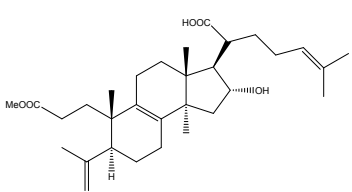

38.Poricoic acid A

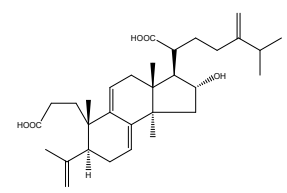

39.Poricoic acid C

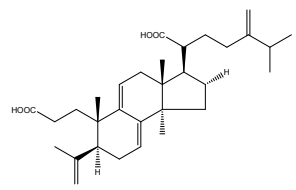

40.Poricoic acid D

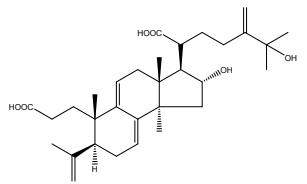


41.Poricoic acid F

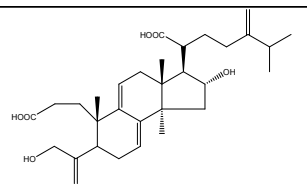

42.Poricoic acid $\mathrm{H}$

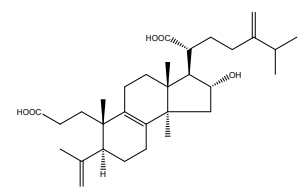

43.Poricoic acid AM

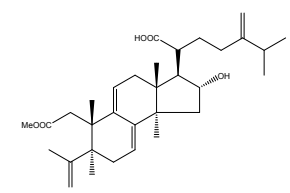

44.Poricoic acid CM

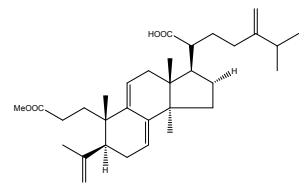

45.Poricoic acid DM

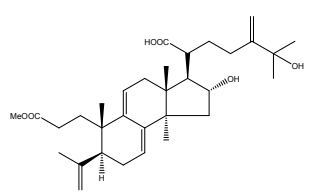

46.Poricoic acid HM

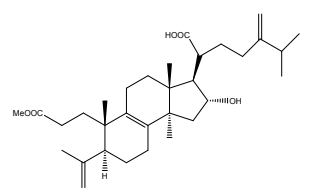

47.6,7-Dehydroporicoic acid H

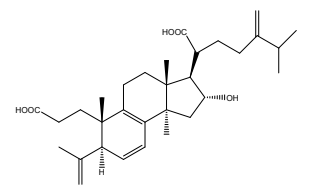

48.25-Hydroxy-poricoic acid C

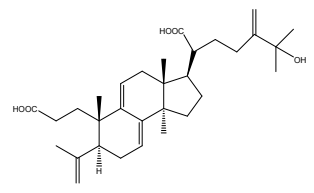


49.25-Hydroxy-poricoic acid $\mathrm{H}$

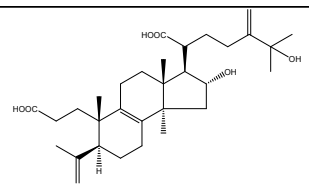

50.26-Hydroxy-poricoic acid DM

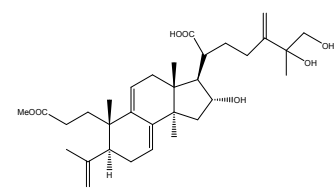

51.25-Methoxyporicoic acid A

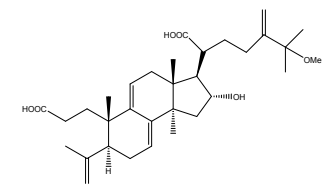

$52.3 \beta$-acetyl-16 $\alpha$-hydroxy dehydrotrametenolic

acid

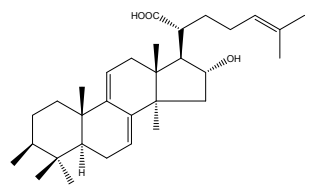

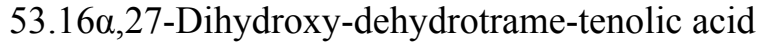

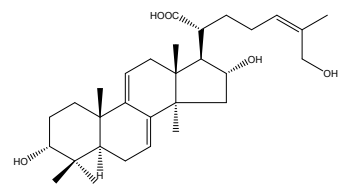

54.pachymic acid methyl ester

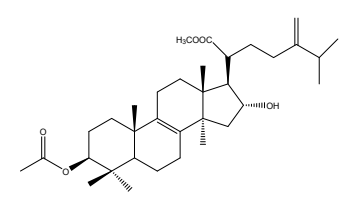

55.16-O-acetyl-pachymic acid

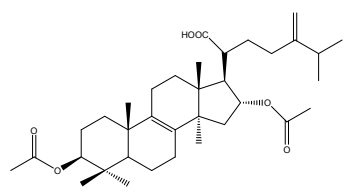

56.16-O-acetyl-pachymicacid methyl ester

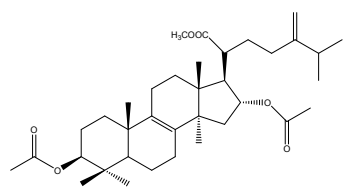


57.31-hydroxyl-16-O-acetyl-pachymic acid

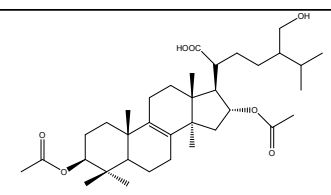

58. Me trametenolate

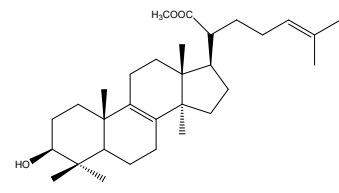

59.acetyl eburicoic acid

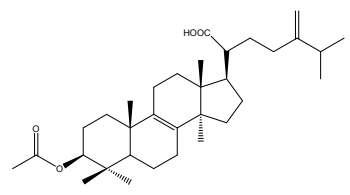

60.16 $\alpha, 25$-dihydroxyeburiconic acid

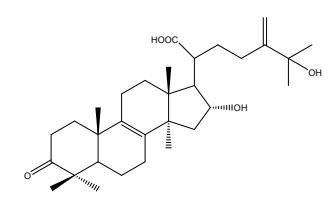

61.6 $\alpha$-羟基-dehydropachymic acid

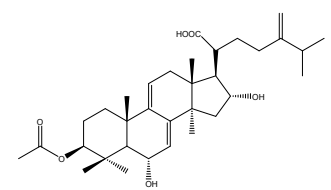

62.33-hydroxy-lanosta-7,9(11),24-trien-21-methyl

ester

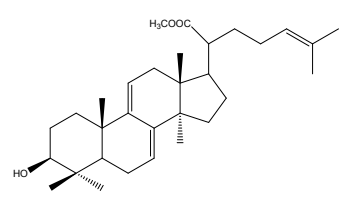

63.3 -hydroxy-16 $\alpha$-acetyl-de-hydrotrametenolic

acid

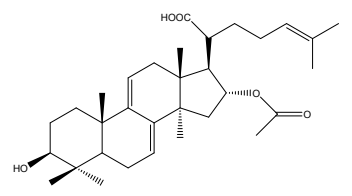

64.3-O-acetyl-dehydroeburicoic acid

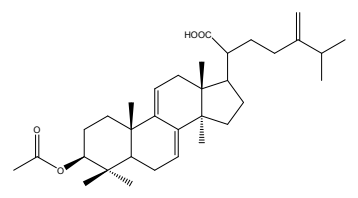


65.Me dehydroeburicoate

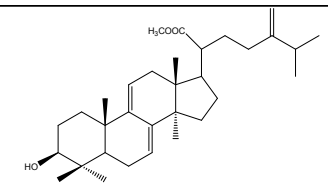

66.25-hydroxyl-3-epi-dehydrotumulosic acid

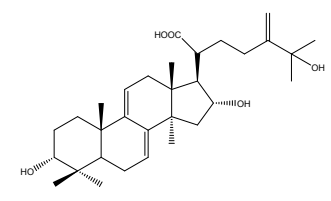

67.6 $\alpha$-hydroxyl-dehydrotumulosic acid

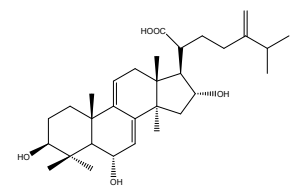

68.29-hydroxyl-dehydrotumulosic acid

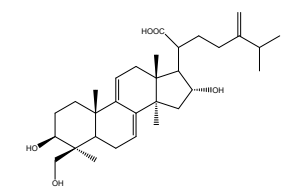

69.29-hydroxyl-dehydropachymic acid

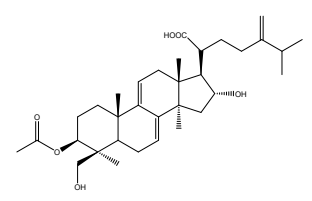

70.3 $\alpha$-benzoyl-dehydrotumulosic acid

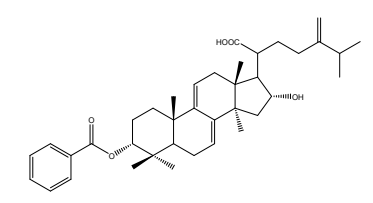

71.3-epi-(3'-O-methyl malonyloxy)-

dehydrotumulosic acid

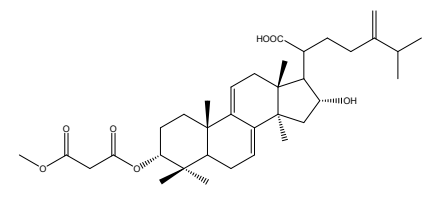

72.3-epi-(3'-hydroxy-3'-methylglutaryloxyl)dehydrotumulosic acid

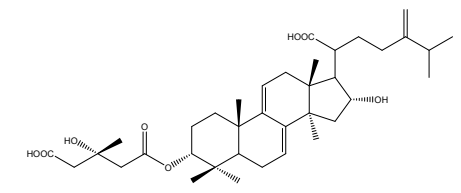


73.25-hydroxyl-polyporenic acid C

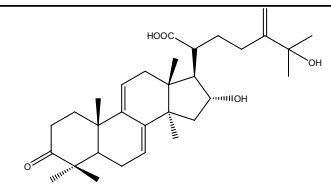

74.poricoic acid AE

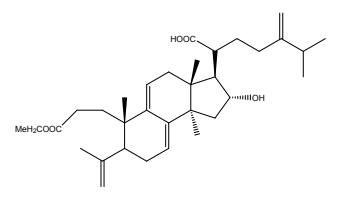

75.poricoic acid CE

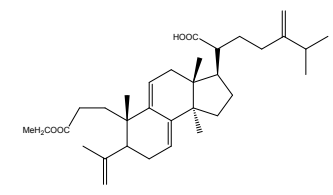

76.3,4-seclanosta-4(28),7,9(11),24-tetraen-3,26-

dioic acid

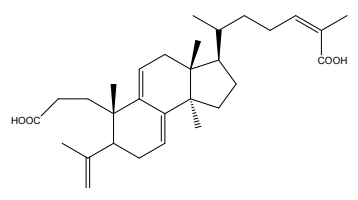

77.Daedaleanic acid A

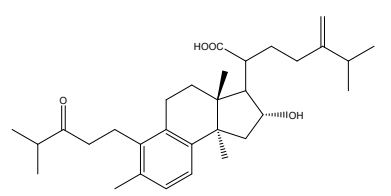

78. $\beta$-amyrin acetate

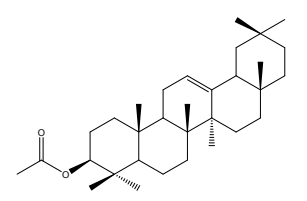

79. $\alpha$-amyrin acetate

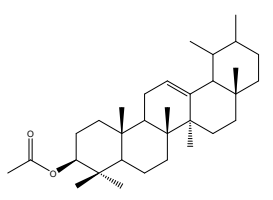

80.oleanic acid

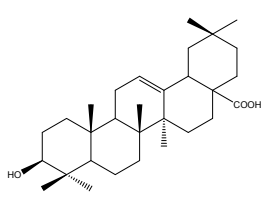


81.oleanic acid 3-acetate

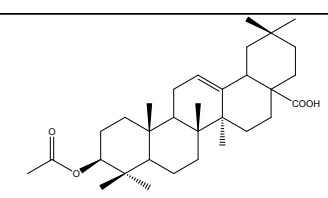

82.Dehydroabietic acid methyl ester

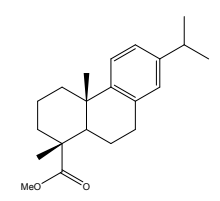

83.7-oxo-15-hydroxydehydroabietic acid

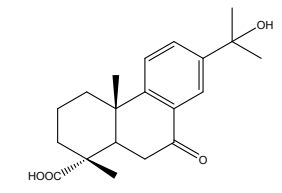

84.ganoderic acid B

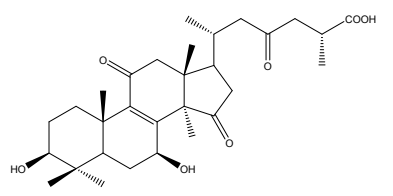

Table S2. Sequences of primers used for reverse transcriptase-PCR. 


\begin{tabular}{ccc}
\hline Gene & Forward primer $\left(5^{\prime}-3^{\prime}\right)$ & Reverse primer $\left(5^{\prime}-3^{\prime}\right)$ \\
\hline iNOS & GGAGTGACGGCAAACATGACT & TCGATGCACAACTGGGTGAAC \\
COX-2 & TTCAACACACTCTATCACTGGC & AGAAGCGTTTGCGGTACTCAT \\
& & \\
TNF- $\alpha$ & CCTGTAGCCCACGTCGTAG & GGGAGTAGACAAGGTACAACCC \\
IL-1 $\beta$ & GAAATGCCACCTTTTGACAGTG & TGGATGCTCTCATCAGGACAG \\
IL-6 & TCTATACCACTTCACAAGTCGGA & GAATTGCCATTGCACAACTCTTT \\
GAPDH & AGGTCGGTGTGAACGGATTTG & TGTAGACCATGTAGTTGAGGTCA \\
\hline
\end{tabular}

Table S3. Cytotoxicity of triterpenoids from Poria cocos on Caco-2 cells. Cell 
viability (\%) was detected by MTT assay. Data are the mean \pm SD from three

independent experiments. ${ }^{*} P<0.05, * * P<0.01$ vs control group.

\begin{tabular}{cccccc}
\hline \multirow{2}{*}{ Compound } & \multicolumn{5}{c}{ Concentration $(\mu \mathrm{M})$} \\
\cline { 2 - 6 } & 10 & 20 & 40 & 60 & 80 \\
\cline { 2 - 6 } HTA & $103.8 \pm 1.6$ & $103.9 \pm 2.3$ & $103.5 \pm 1.6$ & $108.8 \pm 1.7^{* *}$ & $102.1 \pm 1.5$ \\
& & & & \\
DTA & $101.8 \pm 2.1$ & $104.2 \pm 1.8$ & $104.9 \pm 1.4$ & $106.4 \pm 1.7^{*}$ & $98.4 \pm 1.0$ \\
DEA & $103.8 \pm 1.6$ & $104.2 \pm 1.8$ & $102.2 \pm 3.6$ & $102.1 \pm 2.8$ & $95.8 \pm 1.7$ \\
PPAC & $104.7 \pm 2.7$ & $104.5 \pm 7.2$ & $103.9 \pm 1.1$ & $104.1 \pm 1.7$ & $95.4 \pm 2.8$ \\
& & & & & \\
PA & $100.5 \pm 4.1$ & $100.9 \pm 3.9$ & $98.9 \pm 4.0$ & $105.8 \pm 0.7$ & $96.1 \pm 3.3$ \\
DPA & $103.8 \pm 1.6$ & $104.2 \pm 1.8$ & $102.2 \pm 3.6$ & $102.1 \pm 2.8$ & $90.8 \pm 1.2^{* *}$ \\
\hline
\end{tabular}


Table S4. Cytotoxicity of triterpenoids from Poria cocos on 293 T cells. Cell viability (\%) was detected by MTT assay. Data are the mean \pm SD from three independent experiments. ${ }^{*} P<0.05,{ }^{* *} P<0.01$ vs control group.

\begin{tabular}{cccccc}
\hline \multirow{5}{*}{ Compound } & \multicolumn{5}{c}{ Concentration $(\mu \mathrm{M})$} \\
\cline { 2 - 6 } & 10 & 20 & 40 & 60 & 80 \\
\hline \multirow{2}{*}{ HTA } & $99.2 \pm 2.7$ & $97.9 \pm 4.0$ & $102.6 \pm 3.3$ & $101.9 \pm 2.8$ & $86.2 \pm 2.1^{* *}$ \\
& & & & \\
DTA & $101.6 \pm 3.2$ & $99.7 \pm 2.9$ & $103.9 \pm 2.9$ & $102.1 \pm 1.7$ & $96.5 \pm 3.6$ \\
DEA & $103.2 \pm 1.7$ & $104.3 \pm 3.1$ & $96.3 \pm 1.8$ & $96.8 \pm 2.2$ & $92.3 \pm 2.6^{* *}$ \\
PPAC & $98.6 \pm 1.9$ & $102.6 \pm 1.7$ & $97.5 \pm 3.5$ & $104.1 \pm 3.2$ & $85.2 \pm 4.9$ \\
PA & $103.7 \pm 2.8$ & $96.8 \pm 1.3$ & $105.7 \pm 1.6$ & $97.6 \pm 2.3$ & $97.2 \pm 3.3$ \\
DPA & $101.6 \pm 3.8$ & $102.5 \pm 0.9$ & $97.6 \pm 2.2$ & $95.6 \pm 4.3$ & $85.9 \pm 0.7^{* *}$ \\
\hline
\end{tabular}


Table S5. Cytotoxicity of triterpenoids from Poria cocos on RAW 264.7 cells. Cell viability (\%) was detected by MTT assay. Data are the mean \pm SD from three independent experiments. ${ }^{*} P<0.05, * * P<0.01$ vs control group.

\begin{tabular}{cccccc}
\hline & \multicolumn{5}{c}{ Concentration $(\mu \mathrm{M})$} \\
Compound & 10 & 20 & 40 & 60 & 80 \\
\cline { 2 - 6 } & $102.2 \pm 2.1$ & $107.5 \pm 1.4$ & $103.9 \pm 0.7$ & $98.3 \pm 0.8$ & $87.2 \pm 3.2^{* * *}$ \\
HTA & $99.5 \pm 1.7$ & $102.6 \pm 2.5$ & $101.3 \pm 1.6$ & $97.9 \pm 2.2$ & $98.3 \pm 0.7$ \\
DTA & $102.5 \pm 0.9$ & $99.3 \pm 2.5$ & $105.7 \pm 1.4$ & $101.5 \pm 0.7$ & $89.8 \pm 4.2^{*}$ \\
DEA & & & & & \\
PPAC & $98.7 \pm 1.6$ & $103.6 \pm 2.3$ & $101.5 \pm 1.5$ & $95.8 \pm 0.7$ & $90.1 \pm 1.7^{* *}$ \\
PA & $101.4 \pm 2.9$ & $104.2 \pm 2.1$ & $99.6 \pm 1.7$ & $102.1 \pm 1.5$ & $95.5 \pm 2.9$ \\
DPA & $102.6 \pm 2.8$ & $99.2 \pm 1.6$ & $103.2 \pm 1.1$ & $97.3 \pm 2.9$ & $88.2 \pm 2.3^{* *}$ \\
\hline
\end{tabular}


Table S6. Effect of HTA on LPS-induced NO production in macrophages.

Inhibition of NO (\%) and cell viability (\%) were compared to the control group. Each value represents the mean $\pm \mathrm{SD}$ of at least three independent experiments.

\begin{tabular}{lccc}
\hline \multicolumn{4}{c}{ HTA concentration $(\mu \mathrm{M})$} \\
\hline & 20 & 40 & 60 \\
& & & \\
\hline Inhibition of NO (\%) & $10.6 \pm 0.4$ & $32.7 \pm 1.5$ & $59.3 \pm 3.2$ \\
Cell viability $(\%)$ & $107.5 \pm 1.4$ & $103.9 \pm 0.7$ & $98.3 \pm 0.8$ \\
\hline
\end{tabular}

